\title{
Variation in Private Payer Coverage of Rheumatoid Arthritis Drugs
}

\author{
James D. Chambers, PhD; Colby L. Wilkinson, BA; Jordan E. Anderson, BA; \\ and Matthew D. Chenoweth, MPH
}

\begin{abstract}
BACKGROUND: Payers in the United States issue coverage determinations to guide how their enrolled beneficiaries use prescription drugs. Because payers create their own coverage policies, how they cover drugs can vary, which in turn can affect access to care by beneficiaries.
\end{abstract}

OBJECTIVE: To examine how the largest private payers based on membership cover drugs indicated for rheumatoid arthritis and to determine what evidence the payers reported reviewing when formulating their coverage policies.

METHODS: Coverage policies issued by the 10 largest private payers that make their policies publicly available were identified for rheumatoid arthritis drugs. Each coverage determination was compared with the drug's corresponding FDA label and categorized according to the following: (a) consistent with the label, (b) more restrictive than the label, (c) less restrictive than the label, or (d) mixed (i.e., more restrictive than the label in one way but less restrictive in another). Each coverage determination was also compared with the American College of Rheumatology (ACR) 2012 treatment recommendations and categorized using the same relative restrictiveness criteria. The policies were then reviewed to identify the evidence that the payers reported reviewing. The identified evidence was divided into the following 6 categories: randomized controlled trials; other clinical studies (e.g., observational studies); health technology assessments; clinical reviews; cost-effectiveness analyses; and clinical guidelines.

RESULTS: Sixty-nine percent of coverage determinations were more restrictive than the corresponding FDA label; $15 \%$ were consistent; $3 \%$ were less restrictive; and $13 \%$ were mixed. Thirty-four percent of coverage determinations were consistent with the ACR recommendations, $33 \%$ were more restrictive; $17 \%$ were less restrictive; and $17 \%$ were mixed. Payers most often reported reviewing randomized controlled trials for their coverage policies (an average of 2.3 per policy). The payers reported reviewing an average of 1.4 clinical guidelines, 1.1 clinical reviews, 0.8 other clinical studies, and 0.5 technology assessments per policy. Only 1 payer reported reviewing cost-effectiveness analyses. The evidence base that the payers reported reviewing varied in terms of volume and composition.

CONCLUSIONS: Payers most often covered rheumatoid arthritis drugs more restrictively than the corresponding FDA label indication and the ACR treatment recommendations. Payers reported reviewing a varied evidence base in their coverage policies.

J Manag Care Spec Pharm. 2016;22(10):1176-81

Copyright $\odot 2016$, Academy of Managed Care Pharmacy. All rights reserved.

\section{What is already known about this subject}

Private payers in U.S. health care create their own coverage policies for prescription drugs.

Because payers create their own policies, drug coverage policies can vary.

\section{What this study adds}

This study examines variation in the coverage determinations of the largest private payers for rheumatoid arthritis drugs and in the evidence base payers report reviewing when formulating their policies.

Payer coverage of rheumatoid arthritis drugs was typically found to be more restrictive than the drugs' FDA-labeled indications and the American College of Rheumatology 2012 treatment recommendations.

This study found that the evidence base that the included payers reported reviewing in their coverage policies for rheumatoid arthritis drugs varied in terms of volume and composition.

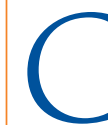

ommercial payers in the United States help guide how their enrolled beneficiary populations use drugs and biologics by developing coverage criteria. Because payers create their own coverage policies, how they cover drugs can vary, which in turn can affect access by beneficiaries.

Payers issue coverage policies to describe the criteria that plan beneficiaries must meet for select drugs to be covered and, in those policies, often report the evidence they reviewed when formulating the coverage determination. A growing body of research is using coverage policies to study trends in how payers cover medical technologies. Researchers have studied trends in Medicare National Coverage Determinations (NCDs), compared determinations for precision medicine technology, examined inconsistencies in Medicare and private payer coverage of medical devices, and evaluated the coverage policies for medical interventions of the largest U.S. commercial payers. ${ }^{1-7}$

This study adds to the literature by evaluating the coverage policies for rheumatoid arthritis drugs of the largest payers. First, we determined the restrictiveness of the coverage policies by benchmarking them to each drug's U.S. Food and Drug Administration (FDA)-approved indication and to the American College of Rheumatology (ACR) recommendations for the treatment of rheumatoid arthritis. ${ }^{8}$ Second, we examined what evidence the payers reported reviewing when formulating their coverage policies.

\section{Methods}

The 10 largest private payers that make coverage policies for drugs publicly available for the treatment of rheumatoid arthritis were identified in terms of covered lives using a 2012 report from 
the National Association of Insurance Commissioners. ${ }^{9}$ Kaiser Permanente was excluded because its coverage policies were not publicly available. Each payer's website was searched for coverage policies pertaining to rheumatoid arthritis treatments. Only coverage policies relevant to the payers' commercial lines of business were included. Included coverage policies were current as of April 1,2015 . We focused this research on rheumatoid arthritis drugs because we found that payers typically provide reliable access to coverage policy documentation for this condition. Drugs included in our sample were biologic disease-modifying antirheumatic drugs (DMARDS), including anti-tumor necrosis factor (anti-TNF) drugs (adalimumab, certolizumab pegol, etanercept, golimumab, and infliximab) and other non-anti-TNF biologic DMARDs (rituximab, anakinra, tocilizumab, abatacept, and tofacitinib).

\section{Comparing Coverage Determinations with Corresponding FDA Labels}

The FDA approved the selected drugs for patients suffering from moderate to severely active rheumatoid arthritis. However, the FDA approvals differed for each drug with respect to whether they were approved for use as monotherapy or for use concurrently with nonbiologic DMARDs, such as methotrexate. The FDA approvals also differed for each drug with respect to whether they were indicated as first-line treatments or whether they required patients to first fail alternative treatments, such as DMARDs.

To compare coverage policies with the corresponding FDA labels, each drug's FDA label was identified using the FDA Approved Drug Products search tool. ${ }^{10}$ Labels were current as of April 1, 2015. The parameters of each payer's coverage decision were compared with the parameters of the FDA label, using the labeled indication, contraindications, and black box safety warnings. Payer coverage for rheumatoid arthritis treatments were categorized as follows: (a) more restrictive than the FDA label (the payer placed more conditions on coverage than the FDA label); (b) consistent with the FDA label; (c) less restrictive than the FDA label (the payer provided coverage for off-label indications not included in the FDA label); or (d) mixed (the payer's coverage policy was more restrictive than the FDA approval in one way but was less restrictive in another). An example of a mixed coverage policy was Blue Cross Blue Shield of Michigan's policy for infliximab. This payer covered infliximab for patients who had first failed treatment with a nonbiologic DMARD—a restriction absent from the FDA approval-but covered the drug for use as monotherapy, that is, its use was not required to be in combination with methotrexate, which was an off-label indication.

When coverage policies were more restrictive than the corresponding FDA labels, they were further categorized as (a) patient restrictions (the payer restricted coverage to patients suffering from disease of particular severity); (b) step therapy restrictions (the payer restricted coverage to patients who had first failed an alternative treatment); or (c) combination restrictions (the payer covered the drug only when used in combination with other drugs).

\section{Comparing Coverage Policies with ACR Recommendations}

Payer coverage policies were also compared with the ACR 2012 recommendations for the treatment of rheumatoid arthritis. ${ }^{8}$ The ACR has since published updated recommendations, but the 2012 recommendations were current as of April 1, 2015. ${ }^{11}$ To summarize the guidelines, for patients suffering from rheumatoid arthritis with moderate to high disease activity, the ACR recommended the following: (a) first-line treatment with methotrexate or combination nonbiologic DMARD therapy; (b) if disease activity remains high, an anti-TNF biologic (adalimumab, certolizumab pegol, etanercept, golimumab, or infliximab), or abatacept or rituximab should either replace the nonbiologic DMARD therapy or be added to it; (c) if disease activity remains high, patients should be switched to another type or category of anti-TNF or non-anti-TNF biologic (including tocilizumab). The ACR 2012 recommendations were published before tofacitinib was approved in November 6, 2012, and did not include anakinra because of the lack of new data and its infrequent use. ${ }^{8}$

Each coverage policy was compared with the guidelines and classified as follows: (a) more restrictive (the payer placed conditions on coverage beyond ACR recommendations); (b) consistent with ACR recommendations; (c) less restrictive (the payer additionally covered the drug for an indication not included in ACR recommendations); or (d) mixed (the payer's coverage policy was more restrictive than ACR recommendations in one way but was less restrictive in another).

\section{Assumptions}

Plan Type. One payer, Cigna, modified its coverage policies depending on whether the drug was offered through an employer group benefit plan or an individual and family benefit plan. For this study, the coverage policy pertaining to the employer group benefit plan was used, since it seemed to be the plan's predominant offering.

Route of Administration. Three drugs in our dataset-abatacept, golimumab, and tocilizumab—can be administered subcutaneously and intravenously. On occasion, payers modified their coverage policies depending on the route of administration. For these drugs, when comparing the coverage policy to the FDA approval, the route of administration for which the payer's coverage policy was least restrictive was considered. The same approach was used when comparing coverage policies with ACR recommendations.

\section{Examining Evidence that Payers Reported Reviewing in Coverage Policies}

Evidence that payers reported reviewing in their coverage policies was examined. Each coverage policy was reviewed, and evidence cited or discussed within the policy that pertained to the relevant drug was identified. The evidence was categorized 
into 6 categories: randomized controlled trials (RCTs); other clinical studies (e.g., nonrandomized controlled trials, prospective cohort studies, and consecutive case series studies); clinical reviews (including systematic reviews of clinical evidence); clinical guidelines; health technology assessments; and costeffectiveness analyses. The frequency that the payers reviewed evidence in each category was then reported.

\section{Results}

We identified 94 coverage policies across the 10 payers. Eight payers issued coverage policies for all 10 drugs; Highmark did not issue coverage policies for 4 of the drugs, and UnitedHealth Group did not issue coverage policies for 2 of the drugs.

\section{Comparing Coverage Policies with Corresponding FDA Approval}

Sixty-nine percent of coverage determinations were more restrictive than the corresponding FDA approvals; 15\% were consistent; 3\% were less restrictive; and 13\% were mixed (Figure 1).

Coverage restrictions were almost exclusively step therapy restrictions ( 76 of 77 restrictions), although the nature of the restriction frequently differed. For instance, while the FDA approved golimumab as a first-line treatment, Humana covered golimumab for patients who had first failed adalimumab and etanercept, while HealthNet covered golimumab for patients who had first failed methotrexate. We found a single combination restriction among the coverage policies. Cigna covered certolizumab pegol in combination with methotrexate, a condition not included in the FDA approval. No coverage determinations included patient restrictions.

\section{Comparing Coverage Policies with ACR Recommendations}

Thirty-four percent of coverage determinations were consistent with ACR recommendations; 33\% were more restrictive; 17\% were less restrictive; and 17\% were mixed (Figure 1). When coverage determinations were more restrictive than the ACR recommendations, it was because they required that patients first fail 1 or more biologic DMARDs. For example, in Highmark's coverage policy for abatacept, patients were required to fail a nonbiologic DMARD and a biologic DMARD before gaining access to the drug, a requirement not included in the ACR recommendations. When coverage determinations were less restrictive than the ACR recommendations, it was typically because they did not require that patients first fail a nonbiologic DMARD.

\section{Comparing Evidence that Payers Reported Reviewing in Coverage Policies}

Health Care Service Corporation was the only payer that did not report the evidence they reviewed in their publicly accessible coverage policies. WellPoint and Aetna reported

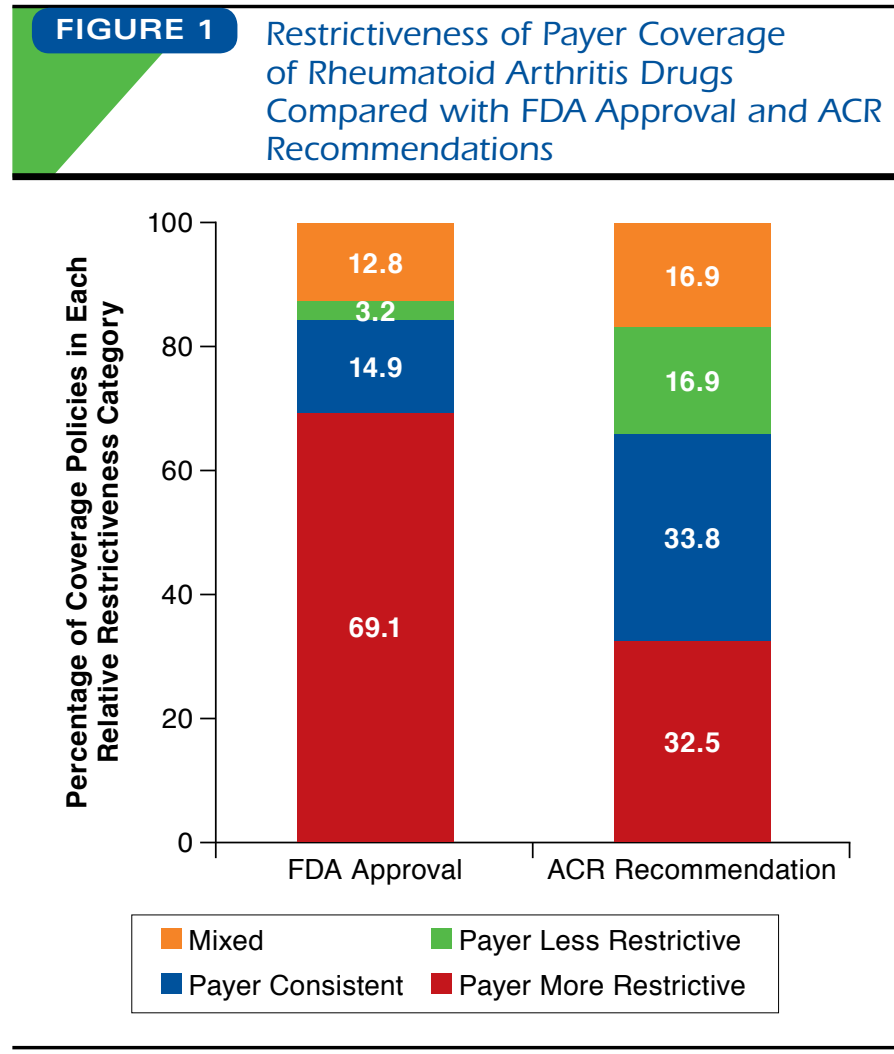

ACR=American College of Rheumatology; FDA = U.S. Food and Drug Administration.

reviewing the largest volume of evidence-an average of 12.4 and 11.9 pieces of evidence per coverage policy, respectively (Figure 2). In contrast, Blue Cross Blue Shield of Michigan and UnitedHealth Group reviewed an average of 2.0 and 1.0 pieces of evidence per coverage policy, respectively.

Of the evidence categories, RCTs most often featured in the evidence that the payers reported reviewing (an average of 2.3 per policy that reported the evidence payers reviewed). WellPoint reviewed the most RCTs (average of 7.4 per policy), while UnitedHealth Group reviewed the fewest (average of 0.1 per policy). Seven payers reported reviewing studies that fell in the "other clinical studies" category (average of 0.8 per policy): WellPoint reported reviewing the most (average of 1.9 per policy), while Humana reported reviewing the fewest (average of 0.3 per policy). Each payer reported reviewing clinical guidelines (average of 1.4 per policy): Cigna reviewed the most (average of 2.7 per policy), while Aetna reported reviewing the fewest (average of 0.7 per policy). Seven payers reported reviewing clinical reviews (average of 1.1 per policy): Aetna reported reviewing the most clinical reviews (average of 4.0 per policy), while UnitedHealth Group reported reviewing the fewest (average of 0.1 per policy). Four payers reported reviewing health technology assessments (average of 0.5 per policy): 


\section{FIGURE 2 Proportion of Each Evidence Category Reviewed in Payer Coverage Policies for} Rheumatoid Arthritis Treatments

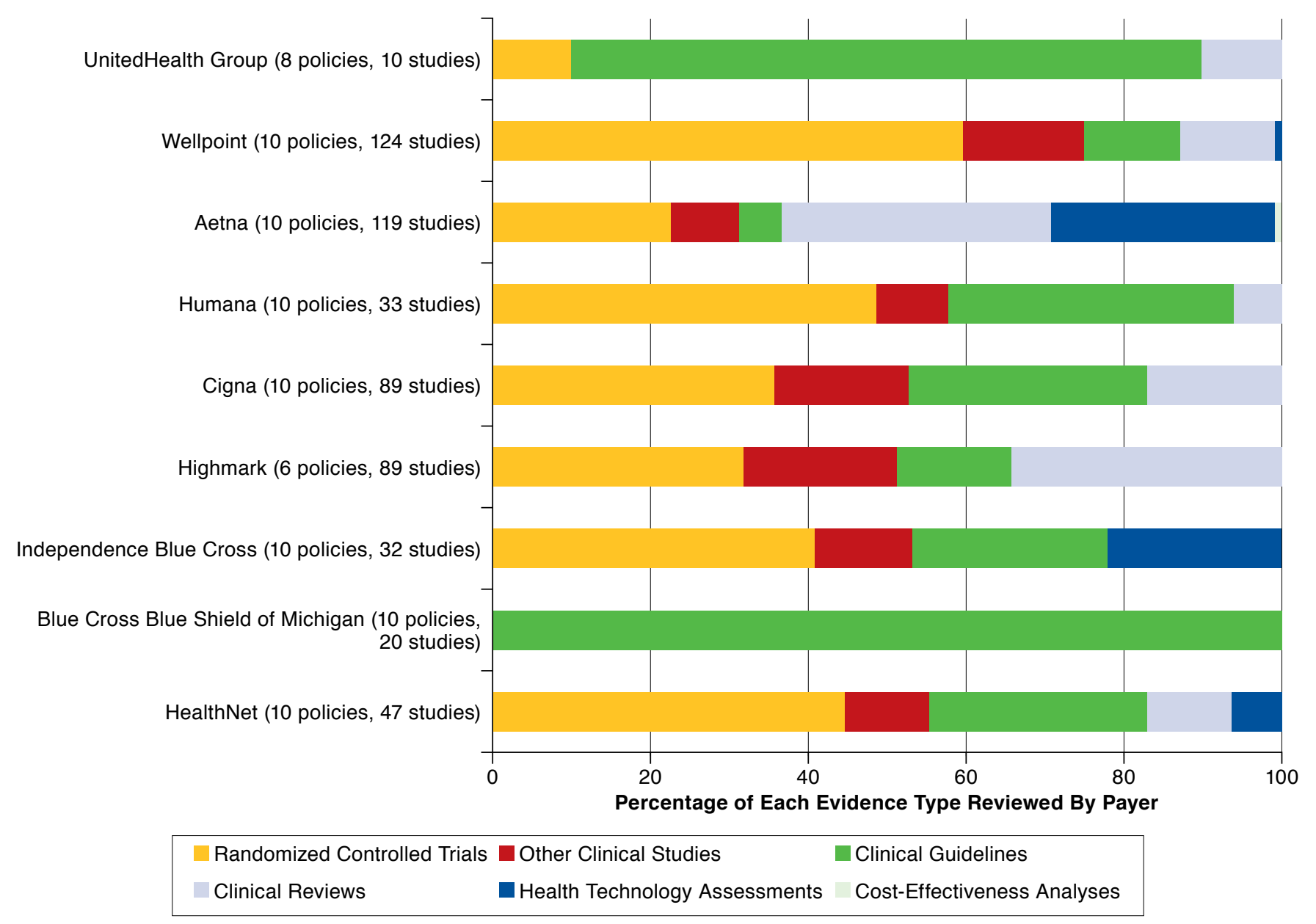

Note: Health Care Service Corporation did not report the evidence it reviewed in its publicly accessible coverage policies.

Aetna reported reviewing the most (average of 3.4 per policy), while WellPoint reported reviewing the fewest (average of 0.1 per policy). Aetna reported reviewing 1 cost-effectiveness analysis in its coverage policies and was the only payer to do so.

\section{Discussion}

A common finding across each aspect of this study was the substantial variation among the payers selected for this study. While all payers covered the drugs that they issued coverage policies for in some way, no drug was covered in the same way by each of the included payers.

Coverage policies for 9 drugs were inconsistent across the selected payers, that is, the drug was covered in a manner consistent with FDA approval by some payers but was covered in a manner that was more or less restrictive by others. The tenth drug, abatacept, was covered more restrictively than the FDA approval by all payers, although the determinations varied. For example, UnitedHealth Group required that patients first fail a nonbiologic DMARD, while Cigna required that patients first fail at least 1 nonbiologic DMARD and 2 biologic DMARDs. Similarly, we found that all payers covered no drug in the same way relative to the ACR recommendations.

The frequency that coverage policies were more restrictive than the corresponding FDA approvals is consistent with the findings of similar research that studied Medicare coverage of medical devices and Part B drugs in NCDs. ${ }^{12}$ These findings follow from the fact that the FDA and payers assess medications using different criteria. ${ }^{13}$ The FDA is charged with ensuring that medications are safe and effective. In contrast, payers cover medications that they determine to be medically necessary. 
Payers must weigh various additional factors into their assessments, including benefit risk trade-offs and, likely, drug cost.

Inconsistencies between payers' coverage determinations and the ACR recommendations are not entirely unexpected given differences in the form they take. While payers make separate judgments for each drug, the ACR generally groups drugs in the same class together in their recommendations (e.g., anti-TNF drugs). Nevertheless, the frequency that payers' coverage determinations were more restrictive than the ACR recommendations was noteworthy.

The variation among payer coverage policies is more difficult to explain, although it is consistent with the findings of research that compared Medicare and private payer coverage of medical devices. ${ }^{6}$ One potential explanation is that payers based their determinations on different evidence. We found that payers reported reviewing different volumes of evidence and different types of evidence with different frequencies. Indeed, Aetna was the only payer that reviewed evidence that fell into all 6 evidence categories. However, an important limitation of this study is that payers may not fully report the evidence base that they reviewed in their coverage policies. A further explanation could be that some payers more heavily weigh drug cost into their decisions than others. While we found that only Aetna explicitly considered relative drug cost, other payers almost certainly weighed drug cost in their determinations in some way. ${ }^{14}$

The varying beneficiary populations may drive variation across payers. It may also be that some payers offer more generous drug coverage to attract enrollees. Finally, the availability of therapeutic alternatives, competing health plan coverage policies, and input from customers (e.g., large employers) may influence decision making. ${ }^{15}$

\section{Limitations}

Some limitations to this study need to be considered. Not all payers issued a coverage policy for each drug. Further, it is unclear how generalizable our findings are to payers not included in this study. We did not account for the appeals process that is typically available when coverage of a drug is denied. As noted, payers may not fully report the evidence base that they review when formulating coverage determinations. Furthermore, it may be that payers perform their own assessments, such as cost-effectiveness analyses, that they do not report in their coverage policies. ${ }^{14}$ Finally, because of sample size, we were unable to examine the consistency of the coverage determinations with the evidence the payers reported reviewing.

\section{Implications}

Our findings indicate that beneficiaries in different health plans may have variable access to rheumatoid arthritis drugs. Inconsistencies in how payers cover drugs increase the complexity of care delivery, since physicians are required to tailor a patient's care to the patient's insurance. However, it is contended that differences in coverage policy will inevitably lead to differences in how drugs are used, which in turn may advance our understanding of how to best use these treatments and, thus, accelerate their appropriate use. ${ }^{16}$

The identified variation in the evidence that payers reported reviewing in their coverage policies suggests challenges for manufacturers when creating clinical development programs for their products. A better understanding of evidence required by payers would aid in the generation of clinical evidence that best meets payer requirements and would expedite patient access to treatments. ${ }^{13,17}$

\section{Conclusions}

Substantial variation was found in how the largest payers cover rheumatoid arthritis treatments and in the evidence that payers reported reviewing in their coverage determinations. How payers covered the rheumatoid arthritis drugs used in this study was typically more restrictive than the corresponding FDA approvals and the ACR recommendations for treatment.

\section{Authors}

JAMES D. CHAMBERS, PhD, Tufts University School of Medicine and Center for the Evaluation of Value and Risk in Health, Institute for Clinical Research and Health Policy Studies, Tufts Medical Center, Boston, Massachusetts. COLBY L. WILKINSON, BA; JORDAN E. ANDERSON, BA; and MATTHEW D. CHENOWETH, $\mathrm{MPH}$, Center for the Evaluation of Value and Risk in Health, Institute for Clinical Research and Health Policy Studies, Tufts Medical Center, Boston, Massachusetts.

AUTHOR CORRESPONDENCE: James D. Chambers, PhD, Center for the Evaluation of Value and Risk in Health, Institute for Clinical Research and Health Policy Studies, Tufts Medical Center, 800 Washington St., \#63, Boston, MA 02111.

E-mail: jchambers@tuftsmedicalcenter.org.

\section{DISCLOSURES}

Funding for this study was provided by Genentech. Chambers has participated in a Sanofi advisory board, unrelated to this study. The authors report no other potential conflicts of interest.

Study concept and design were contributed by Chambers. Anderson, Wilkinson, and Chenoweth collected the data, assisted by Chambers, and data interpretation was primarily performed by Chambers, along with Anderson and with assistance from Wilkinson and Chenoweth. The manuscript was written primarily by Chambers, along with Wilkinson and with assistance from Anderson and Chenoweth. Chambers, Chenoweth, Wilkinson, and Anderson revised the manuscript. 


\section{REFERENCES}

1. Chambers JD, Chenoweth MD, Pyo J, Cangelosi MJ, Neumann PJ. Changing face of Medicare's National Coverage Determinations for technology. Int J Technol Assess Health Care. 2015;31(5):347-54.

2. Chambers JD, Chenoweth M, Cangelosi MJ, Pyo J, Cohen JT, Neumann PJ. Medicare is scrutinizing evidence more tightly for national coverage determinations. Health Aff (Millwood). 2015;34(2):253-60.

3. Meckley LM, Neumann PJ. Personalized medicine: factors influencing reimbursement. Health Policy. 2010;94(2):91-100.

4. Hresko A, Haga SB. Insurance coverage policies for personalized medicine. J Pers Med. 2012;2(4):201-16.

5. Chambers JD, Morris S, Neumann PJ, Buxton MJ. Factors predicting Medicare national coverage: an empirical analysis. Med Care. 2012;50(3):249-56

6. Chambers JD, Chenoweth M, Thorat T, Neumann PJ. Private payers disagree with Medicare over medical device coverage about half the time. Health Aff (Millwood). 2015;34(8):1376-82.

7. Chambers JD, Chenoweth M, Neumann PJ. Mapping US commercial payers' coverage policies for medical interventions. Am J Manag Care. Forthcoming 2016

8. Singh JA, Furst DE, Bharat A, et al. 2012 update of the 2008 American College of Rheumatology recommendations for the use of disease-modifying antirheumatic drugs and biologic agents in the treatment of rheumatoid arthritis. Arthritis Care Res (Hoboken). 2012;64(5):625-39.
9. National Association of Insurance Commissioners. 2012 Accident and Health Policy Experience Report. Kansas City, MO: National Association of Insurance Commissioners; 2013. Available at: http://www.naic.org/prod_ serv/AHP-LR-13.pdf. Accessed September 9, 2016.

10. U.S. Food and Drug Administration. Drugs@FDA. FDA approved drug products. Available at: https://www.accessdata.fda.gov/scripts/cder/drugsatfda/. Accessed September 8, 2016.

11. Singh JA, Saag KG, Bridges SL Jr. et al. 2015 American College of Rheumatology guideline for the treatment of rheumatoid arthritis. Arthritis Rheumatol. 2016;68(1):1-26.

12. Chambers JD, May KE, Neumann PJ. Medicare covers the majority of FDA-approved devices and Part B drugs, but restrictions and discrepancies remain. Health Aff (Millwood). 2013;32(6):1109-15.

13. Neumann PJ, Chambers JD. Medicare's enduring struggle to define "reasonable and necessary" care. N Engl J Med. 2012;367(19):1775-77.

14. Chambers JD. Do changes in drug coverage policy point to an increased role for cost-effectiveness analysis in the USA? Pharmacoeconomics. 2014;32(8):729-33.

15. Dranove D, Hughes EF, Shanley M. Determinants of HMO formulary adoption decisions. Health Serv Res. 2003;38(1 Pt 1):169-90.

16. Bailey PG. Medicare and national coverage. Health Aff (Millwood). 2005;24(1):295-96.

17. Dean BB, Ko KJ, Graff JS, Localio AR, Wade R, Dubois RW. Transparency in evidence evaluation and formulary decision-making: from conceptual development to real-world implementation. P T. 2013;38(8):465-83. 